\title{
MATERIALES
}

MATERIALS

\section{El Bloque de Tierra Comprimido o BTC Una alternativa de Construcción para la Arquitectura Contemporánea}

\author{
The Compressed Ground Block or BTC \\ A Construction Alternative for Contemporary Architecture
}

Darío Ernesto Angulo Jaramillo* y Ana Katherine Carreño Charry***

Citar este artículo como: Angulo Jaramillo, Darío Ernesto y Carreño Charry, Ana Katherine (2017). El Bloque de Tierra Comprimido o (BTC).

Una alternativa de Construcción para la Arquitectura Contemporánea. Revista Nodo, 12(23), 31-37

\section{Resumen}

La degradación del planeta ha llevado tanto a los organismos de investigación y experimentación, como a las empresas privadas y particulares a plantear nuevas y mejores alternativas de construcción que minimicen el impacto ambiental negativo para la fauna, la flora y recursos naturales en general. Muchas de estas alternativas retoman sistemas constructivos vernáculos y materiales amigables con la naturaleza, como por ejemplo la tierra cruda para la construcción.

Es por esto que el bloque de tierra comprimido o BTC es unas de las técnicas de construcción más representativas en tierra cruda, debido a su posibilidad de industrialización y producción a gran escala de manera rápida y económica, que lo hace una alternativa viable, durable y sostenible para todo tipo de obra ya que se comporta como un prefabricado tradicional con la diferencia de que no produce $\mathrm{CO}_{2}$; por ello en este artículo se pretende mostrar las bondades de este material y situarlo en el contexto de la realidad actual.

Palabras clave: Tierra cruda, Arquitectura contemporánea, Bloque de tierra comprimido, Alternativas amigables, sostenibilidad.

\section{Abstract}

The degradation of the planet has led both research and experimentation organizations, as well as private and private companies to propose new and better construction alternatives that minimize the negative environmental impact for fauna, flora and natural resources in general. Many of these alternatives take up vernacular building systems and materials that are friendly to nature, such as land for construction.

This is why the compressed earth block or BTC is one of the most representative construction techniques in land, due to its possibility of industrialization and large-scale production in a fast and economic way; what makes it a viable, durable and sustainable alternative for all types of work as it behaves like a traditional prefabricated one with the difference that it does not produce $\mathrm{CO}_{2}$, that is why this article aims to show the benefits of this material and contextualize it in the present.

Keywords: Raw land, contemporary architecture, block compressed, earth friendly alternatives, sustainability.

\section{Fecha de recepción: 17-06-2017 • Fecha de aceptación: 14-11-2017}

\footnotetext{
* Arquitecto, miembro hace 25 años de CraTerre- EAG. Correo electrónico: darioangulo@tierratec.com

* Arquitecta con énfasis en urbanismo, patrimonio y trabajo social de la Universidad de La Salle. Correo electrónico: kathycarreno2012@gmail.com
} 


\section{A manera de introducción}

Desde hace aproximadamente 10.000 años, más de la mitad de los habitantes de la tierra viven en construcciones hechas en tierra cruda, las cuales en su mayoría son construidas por ellos mismos. Sin embargo, gracias al rápido avance tecnológico, la globalización, la industrialización y transformación de los territorios, la tierra como material se ha ido desprestigiando y por lo tanto reemplazando por materiales "modernos" altamente contaminantes como lo son el concreto, acero, cemento, bloques de arcilla cocida, etc...

Sin embargo, a partir del siglo XIX con la crisis energética, la crítica a la industrialización y la cumbre ambiental de Río de Janeiro en 1992, aparece un nuevo movimiento de arquitectura sostenible que busca retomar algunos aspectos importantes de la arquitectura vernácula como son el perdurar en el tiempo, permitir el crecimiento amigable y la evolución de los proyectos al tiempo que se inscribe de manera armónica en el medio ambiente y el clima, para producir beneficios ambientales al exigir eficiencia energética en los proyectos y conservación de los recursos naturales; así como también importantes contribuciones al desarrollo de un futuro sostenible. Lo anterior se logra mediante la aplicación de estrategias bioclimáticas que buscan lograr las mejores condiciones de confort dentro y fuera de las construcciones, sin recurrir al uso de sistemas de climatización artificial.

Es por esto que hoy en día las técnicas y materiales usados desde épocas remotas como la construcción en tierra comienza a tener un gran protagonismo en la arquitectura contemporánea, dada a la tendencia de utilizar materiales no contaminantes en la construcción; además el barro cumple eficientemente con muchos conceptos de la arquitectura sostenible ya que no genera contaminación ambiental, no contiene sustancias tóxicas, necesita muy poca energía para su producción y es un aislante termoacústico natural. Sin embargo, aún existen muchos tabúes acerca de la tierra como material ya que si bien fue utilizada por nuestros antepasados también fue prácticamente abandonada con la industrialización; es por esto que hoy en día es necesario enseñar acerca de dicho material pues sobre él hay muchas inquietudes a nivel socioeconómico, cultural y medio ambiental y más aún en estos momentos en que, desafortunadamente, muchas familias están lejos de acceder a una vivienda digna y nuestro patrimonio vernáculo está en peligro de desaparecer.

La pertinencia y la vigencia de la utilización de la tierra está justificada por su disponibilidad, por la adaptación al entorno y por corresponder a una tradición constructiva de muchos pueblos creada y mejorada durante siglos. Y ello es un factor fundamental a la hora de proponer respuestas que deben ser escuchadas y ponerse en práctica para favorecer un desarrollo local sostenible. No obstante, a pesar de que hoy en día estamos volviendo la vista hacia estos materiales, parece que las escuelas universitarias los han dejado de lado en favor de los materiales y técnicas de construcción industrializados. La tierra no forma parte de los temarios docentes y cuando está presente es sólo de una manera testimonial; ocasionalmente se encuentran materias o cursos optativos que posibilita aprender acerca de este material, pero son casos excepcionales.

Es por esto que el camino para la capacitación sobre este tema en nuestro país, está de la mano de profesionales que han tenido la oportunidad de formarse en escuelas o con expertos de otros países en donde la tierra está recuperando su papel. En naciones como Francia, desde hace 37 años la escuela de arquitectura de Grenoble capacita a profesionales en el laboratorio CRAterre, único centro internacional de investigación especializado en construcción en tierra que ha estado trabajando por el reconocimiento de ésta como respuesta a los desafíos relacionados con el medio ambiente, la diversidad cultural y la lucha contra la pobreza.

CRAterre reúne a investigadores, profesionales y profesores para crear herramientas como, por ejemplo, la maleta Pedagogía, un instrumento 
que recoge una serie de experiencias científicas de fácil comprensión con las que las personas interesadas pueden conocer el fascinante mundo de la tierra como material de construcción que permite establecer vínculos creativos entre investigación, acciones de campo, capacitación y difusión de conocimiento. En Colombia actualmente existe tres módulos de esta maleta que es gestionada por la empresa tierraTEC. Además de lo anterior, CRAterre apoya iniciativas a nivel mundial entre las cuales se encuentra Amàco un centro de investigación y experimentación que pretende mejorar, de manera sensible y poética, las materias primas más comunes, como arena, tierra, agua, madera, paja, etc.

Todo ello implica una pedagogía que utiliza las ciencias de la materia, la experimentación, la emoción y la creatividad para favorecer el surgimiento de nuevas prácticas de construcción en armonía con el ser humano y el territorio, lo cual también entraña una colaboración entre investigadores, profesores y profesionales de la construcción en los campos de la arquitectura, la ingeniería, el arte y el diseño. Esta y otras iniciativas invitan a nuestro país a incentivar la formación en este tipo de técnicas para lograr un uso adecuado de los recursos locales, humanos y naturales, mejorar las condiciones de vivienda y de vida de nuestra población y consolidar la diversidad cultural.

En este artículo se hablará en particular del BTC ya que viene directamente de tradiciones ancestrales de construcción con tierra, y continúa progresando conforme a las nuevas generaciones de materiales, pues se considera una alternativa verde, ecológica y económica.

\section{El BTC en la construcción de vivienda sostenible y confortable}

A nivel general, en la actualidad se reconocen 12 técnicas de construcción en tierra sin contar con todas sus variaciones. Entre las más importantes que existen en nuestro país está el bahareque, la tapia pisada, el adobe y el Bloque de Tierra Compri-

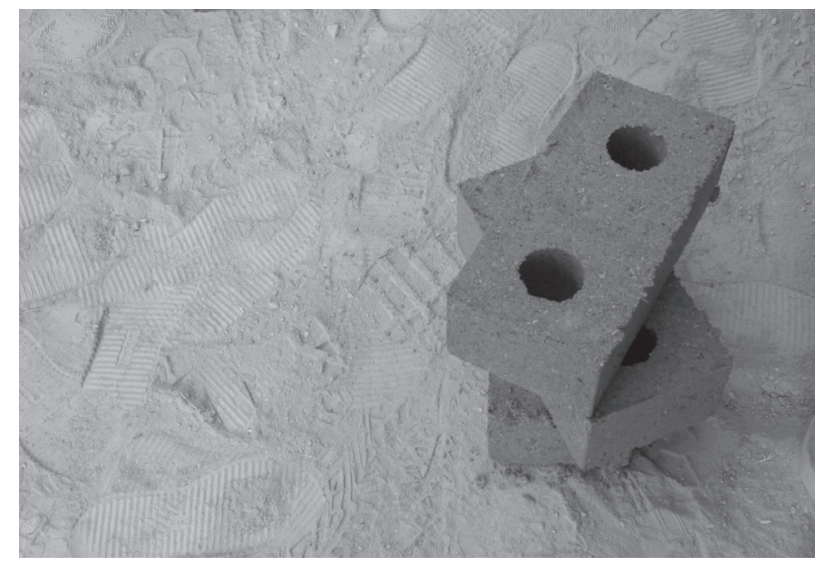

Figura 1. Bloque de tierra comprimido. (2017). Fuente: archivo TierraTEC

mido (en adelante BTC) y con estas técnicas ha sido construido el $90 \%$ del patrimonio vernáculo de nuestro país.

El BTC es un elemento prefabricado de construcción hecho de una mezcla de tierra arcillosa, arena, agregados y estabilizantes compactados individualmente en forma de bloques, utilizando una máquina de compresión. Esta industria apareció en Colombia a comienzos de los años cincuenta gracias el ingeniero Raúl Ramírez (RAM) del Centro Interamericano de Vivienda y Planeamiento -CINVA- dentro del Proyecto 22 de la OEA, como una alternativa económica de material de construcción que actualmente está considerada como una de las tecnologías latinoamericanas más difundidas en el mundo. Su evolución ha permitido que se construyan muchas casas y edificios utilizando BTC, que sigue ganando popularidad en diversos países de Latinoamérica, Europa y África.

El BTC tiene tanto ventajas como desventajas. Dentro de las primeras merece la pena mencionar las siguientes:

- Disponibilidad: la tierra es un material que siempre está disponible y se encuentra fácilmente, sin embargo, es necesario que tenga una calidad específica la cual no es difícil de encontrar.

- Producción: su proceso de producción es simple y tiene posibilidades de industrializa- 


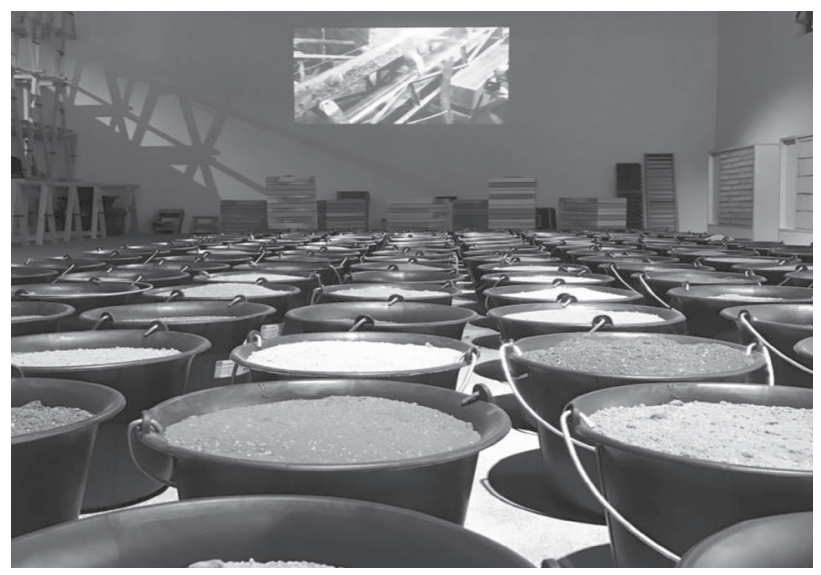

Figura 2. Worskhop "Grounded Material" ETH -Zurich (2015). Fuente: fan page Amàco

ción y producción a gran escala en muy corto tiempo y de una manera más rápida y económica. Además permite tener una unidad de producción móvil que se adapta a toda clase de proyectos (a pequeña, mediana o gran escala) sin generar daño alguno al lugar donde se asienta.

- Inercia térmica: sin duda es una de las mayores ventajas de este material pues la capacidad de almacenar energía dentro de su estructura para restituirla más tarde, lo hace adecuado para ser utilizado con éxito en diferentes condiciones climáticas. En las tierras frías un muro de 30 centímetros de espesor con un peso de $400 \mathrm{Kg}$. $/ \mathrm{m} 2$, tiene la capacidad de producir un desfase térmico de 10 horas; algunas horas después de ocultarse el sol los muros empiezan a restituir el calor ganado durante el día. Este proceso dura 10 horas y garantiza agradables temperaturas al interior de la construcción. Por otra parte, su bajo coeficiente de transmisión térmica, $\mathrm{K}=$ $0.81 \mathrm{wm} 2^{\circ} \mathrm{C}$ lo hace, en cierto modo, térmicamente aislante y evita que el calor acumulado al interior se pierda fácilmente a través de los muros.

En la medida en que los climas se hacen más cálidos se debe aprovechar estas características benévolas del BTC, reduciendo el espesor de los muros para disminuir la capacidad de resti- tución de la inercia del material. Un muro de un espesor de 14 centímetros, con un peso de $195 \mathrm{Kg}$. /m2 y un coeficiente térmico de 1.04 $\mathrm{wm} 2{ }^{\circ} \mathrm{C}$ tiene un tiempo de restitución menor, asegurando así las condiciones de confort durante las horas frescas del amanecer. En las tierras calientes debemos utilizar construcciones de baja inercia térmica con un corto tiempo de restitución y asegurar noches frescas. En ese sentido se debe utilizar entonces bloques de tierra comprimido huecos de baja densidad y cuyo coeficiente de transmisión los conviertan en elementos aislantes que proteja de la inclemencia del sol durante el día.

- Salud: la tierra regula de forma natural el ambiente, no emite gases ni sustancias peligrosas al ambiente, neutraliza el humo del tabaco, evita la formación de hongos y debido a que los BTC no están cocidos conservan sus propiedades únicas de regulación de humedad y acumulación de calor, es decir, el muro absorbe la humedad cuando el tiempo es húmedo y la libera cuando el aire es seco. El peso de los bloques de tierra comprimida hace que un muro de arcilla pueda almacenar bien el calor y luego liberarlo para evitar los cambios de temperatura, por lo que en este tipo de construcciones las personas podrán disfrutar de aire fresco, en especial si tienen problemas respiratorios.

- Medio ambiente: la producción de un m3 de BTC sólo produce 0.114 Ton de CO2 ya que se estabiliza con un mínimo porcentaje de cemento o cal, mientras que un $\mathrm{m} 3$ de ladrillo cocido emite 2,5 Ton de $\mathrm{CO} 2$ y un $\mathrm{m} 3$ de bloque de concreto emite 2,9 Ton de $\mathrm{CO} 2$, etc.; además, solo se requiere un $1 \%$ de energía para producir un bloque y no se producen desperdicios en su fabricación.

- Norma: a diferencia de las demás técnicas de construcción en tierra, en Colombia únicamente el bahareque encementado y el BTC están inscritos en la norma técnica de sismo resistencia NSR 10, en la norma ICONTEC 5324 (bloques de tierra comprimidos para muros 
y divisiones), idéntica a la norma AFNOR XP 13-901 desarrollada por Craterre en Francia; aunque países como Perú, España, Francia, e India, por ejemplo, tienen normas propias para sus técnicas construcción en tierra.

- Propiedades acústicas: garantiza una reducción de los niveles de la presión sonora de 40 decibeles con un muro de un espesor de 20 centímetros.

- Propiedades eléctricas, magnéticas y radiación: la arcilla no se carga de electricidad estática, neutraliza la radiación terrestre y cósmica, no se ve afectada por los campos magnéticos estáticos y es insensible a los campos electromagnéticos. Por lo tanto, según el Instituto Holandés de Biología y Ecología de la Construcción es una buena opción desde el punto de vista del medio ambiente y la salud.

- Aspectos estéticos: el formato de los bloques, su textura, el ambiente natural y la incidencia de la luz aportan un valor adicional.

- Aplicabilidad y diseño: su aplicación es simple y se puede acomodar a diversas formas de arquitectura, por lo que es perfecto para nuevas construcciones, reformas y restauraciones de edificaciones en tierra antiguas.

- Economía: se aprecia una disminución de $\$$ 8.000 a $\$ 25.000$ pesos sin contar con el ahorro de energía a largo plazo; y si no utiliza ningún estabilizante se observará también una reducción en los costos.

- Resistencia: cumpley puede sobrepasar la resistencia exigida por la norma la cual es de $4 \mathrm{Mpa}$ a la compresión y tiene notables propiedades de resistencia al fuego y al impacto balístico; sin embargo, no es un bloque estructural pues la exigencia mínima de este es de $12 \mathrm{Mpa}$ a la compresión.

- Mantenimiento: es vulnerable al impacto del agua y el viento; no obstante con un buen diseño, un pañete y/o hidrófugo se protege y no será necesario un mantenimiento seguido y costoso.
Por otra parte merece la pena mencionar que el BTC se caracteriza por ser un material ambientalmente amigable pues en su proceso de fabricación y transporte no se invierte prácticamente energía, es reciclable y térmicamente muy adaptable a nuestras condiciones climáticas. Nuestra arquitectura vernácula siempre ha sabido utilizar con sabiduría la tierra cruda para adaptar las construcciones al clima y generar las mejores condiciones interiores de temperatura y el BTC conserva las mismas características termo físicas mejorando sus capacidades estructurales y de resistencia a la inclemencia de los factores climáticos.

\section{A manera de conclusión}

Es importante que en Colombia y el mundo las miradas se enfoquen en torno a sistemas constructivos más competitivos, económicos y confortables que establezcan el equilibrio ecológico y permitan la dignificación de la vivienda y de las personas que las habitan, cosa que hasta el momento los insumos industriales no han conseguido; al contrario, éstos han contribuido de una manera importante a la degradación del planeta por su alto poder contaminante y la emisión de gases de efecto invernadero.

El BTC es uno de los métodos de construcción en tierra más aceptado por su parecido con los otros

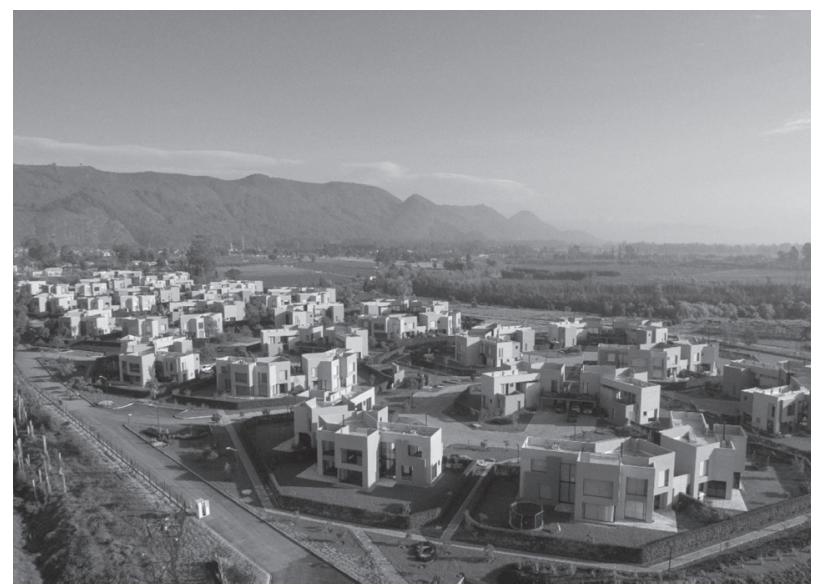

Figura 3. Conjunto Arborada de Cota. (2016). Proyecto seleccionado en el concurso Terra AWARD 2016. Fuente: archivo TierraTEC 


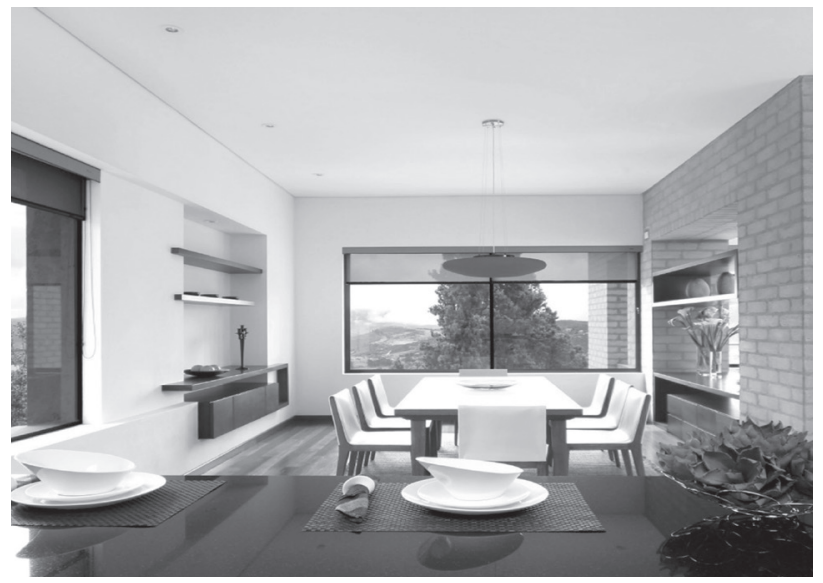

Figura 4. Interior del Conjunto Arboretto (2015). Fuente: archivo TierraTEC

insumos cocidos o de cemento y ser un prefabricado pero con un gran atributo más: no contamina ni en su proceso productivo, ni en el constructivo como sus similares. Es una industria pulcra, con un producto limpio para construir unas edificaciones más sanas y confortables. Como un ejemplo de ello se puede mencionar a tierraTEC, industria colombiana y miembro institucional desde hace 30 años de CRAterre, que no solo produce y distribuye BTC, sino que también acompaña y asesora a sus clientes y brinda a personas con sensibilidad al material, formación en las principales técnicas de construcción en tierra y la maleta pedagógica desarrollada por CRAterre, constituyéndose en una empresa que actualmente lidera en Colombia esta iniciativa por medio de una industria con producción, formación y ventas crecientes.

Por último vale la pena mencionar que la tierra ha conquistado el campo de la arquitectura contemporánea en cientos de proyectos de una gran calidad técnica y estética que emergen en los cinco continentes. El desarrollo de este material con un bajo consumo energético, ampliamente disponible sobre todo el planeta y muy adecuado para obras participativas, podría cubrir una gran parte de las necesidades de viviendas ecológicas y económicas.

Para favorecer un rápido reconocimiento de estas construcciones ejemplares dentro de los profe-

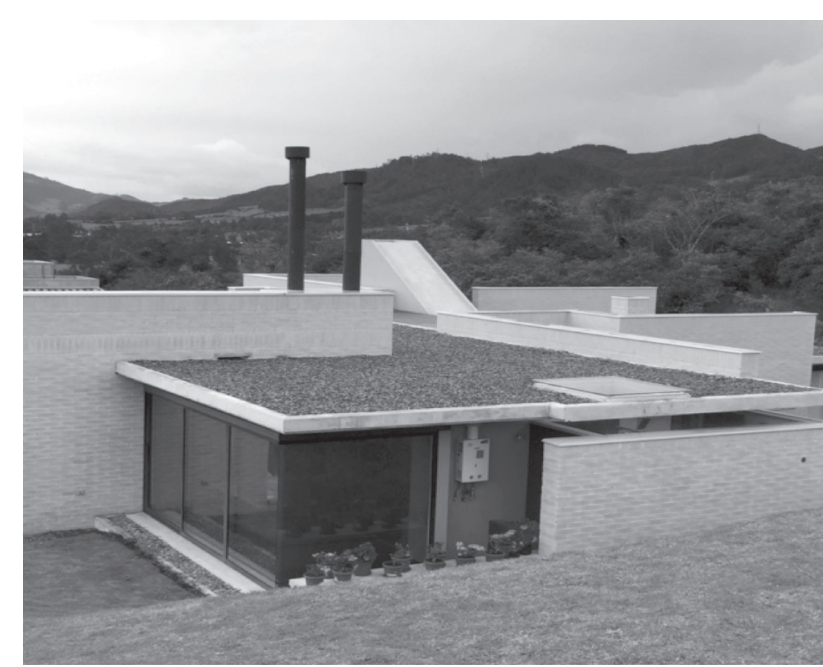

Figura 5. Casa del Arq. Ricardo Leguizamo. (2013). Fuente: archivo TierraTEC

sionales y el gran público es necesario difundirlas valorizando a todos los actores. Bajo el auspicio de la Cátedra UNESCO "Arquitectura de tierra, culturas constructivas y desarrollo sostenible" el laboratorio CRAterre-AE\&CC-ENSAG, Amàco, Les Grands Ateliers, y la revista EcologiK /EK han tomado la iniciativa de lanzar el TERRA Award, primer premio mundial de arquitectura contemporánea en tierra cruda, cuyo objetivo es identificar y distinguir proyectos remarcables buscando enfatizar el valor de los promotores que han elegido la tierra como material, la creatividad de los diseñadores y las habilidades de los constructores y de las empresas. Entre éstos en Colombia se destaca el "Conjunto Arborada de Cota" junto a tierraTEC, elaborado con BTC que fue escogido en el año 2016 como proyecto ejemplo a nivel internacional, demostrando así que es posible retomar técnicas ancestrales de construcción y adaptarlas a la realidad de nuestros días de manera consciente en beneficio no solo del planeta sino también de quienes lo habitan.

\section{Referencias bibliográficas}

\author{
Carazas, W.; Rivero, A. (2003). Tratado de la cons- \\ trucción en tierra, Vol. 1 Ediciones Ediciones \\ Parenthese. Francia. CraTerre- EAG.
}


Doat, P.; Hays, A.; Houben, H., Matuk, S.; Vitoux F. (1979). Construire en terre. Francia. CraTerreEAG.

Rigassi, V. (1995). El bloque de tierra comprimido, Vol. 1 Manual de producción. Francia. CraTerre- EAG.

Rigassi, V. (1995). El bloque de tierra comprimido, Vol. 2 Manual de concepción y producción. Francia. CraTerre- EAG.

Stulz, Roland; Mukerji, Kiran. (1981). Materiales de construcción apropiados. Suiza. IT Publicaciones.
Gazin-Müller, D. (2015). La arquitectura de hoy en día. Museo / CRAterre en partenariat avec amàco.

Gauzin-Müller, D. (2010). comment intégrer une approche éco-responsable?. Enseignement de l'archi-tecture en France.

Doat, P., Guillaud, H., Joffroy, T. \& rakotomamonjy, B. (2011). Demande d'habilitation du diplôme de spécialisation et d'approfondissement en architecture, DSAArchitecture de Terre. 1. CRA Terre-ENSAG. 